\title{
Precision, Speed, and Distraction in Time-Limit Tests
}

\author{
W. M. Pleters and Ad H. G. S. van dor Ven \\ University of thimegen
}

Three models are presented that account for the effects of speed and precision in mental test scores as measured by time-limit intelligence tests. To estimate precision, it is shown that the Rasch modeland, as a special case, the binomial error model - can be used, focusing on the number of items correct and the number of items attempted (usually considered as estimators of these effects). To estimate speed, a model called the Poisson-Erlang model is presented. It is shown that speed can be analyzed into two components: processing time and distraction time. Parameters of the Poisson-Erlang model are used to estimate these components. An outline is given of a new method for analyzing time-limit tests, combining the different models. In addition, it is shown that the Poisson-Erlang model can be used to account for response latencies commonly obseryed in mental concentration tests.

Several authors (e.g., Furneaux, 1961; Guilford, 1959; Spearman, 1927; Thurstone, 1937) have stressed the importance of precision, speed, and distraction as the main determinants of mental test performance. Spearman (1927) has stated:

Now, if we desire any genuine measurement of cognitive ability, it is to these universal quantitative properties of clearness [precision] and speed that we are obliged to turn. (p. 245)

Spearman al so recognized the importance of distraction in mental tests:

Here in oscillation, then, we have come upon a new single and universal factor, a third in addition to $g$ and perseveration. To account for it, one might not unnaturally turn to those writers who have all along been attributing the phenomenon to a central and therefore presumably single influence. But as to the nature of this advocated central influence, and the reason for its producing oscillation, few of these writers have even attempted any explanation-beyond giving to it the not very illuminating title of 'attention' or 'apperception.' (Spearman, 1927, p. 326)

Guilford pointed at the importance of speed, relating it to the number of items attempted:

From a number of sources we have suggestions from time to time that there are mental-speed factors involved in tests of intellectual qualities. Some say that there is a general-speed factor in all tests that have some degree of speeding involved. This is best shown when the scores analyzed are in terms of number of items attempted, overlooking errors. (Guilford, 1959, p. 398)

However, none of these authors has indicated how the observed score, i.e., the number of items correct, is determined by these factors or how they are to be measured.

APPLIED PSYCHOLOGICAL MEASUREMENT

Vol.6. No. 1. Winter 1982, pp. 93-109

(C) Copyright 1982 Applied Psychological Measurement Inc.

0146-6216/82/010093-17\$1.85 
This paper proposes a theory and three models that provide a means to test the effects of these factors in the mental test score. In addition, a review of research on intelligence tests is presented, the central issue being, What is measured in time-limit intelligence tests?

A tentative conclusion of this research is that the (standardized) number of items correct score used in some intelligence tests is a composite score, determined by four factors: two mental processing factors, speed and precision, and two mental concentration factors, average dur ation of distraction and average frequency of occurrence of distraction.

First, some models for speed and precision will be discussed, followed by a theory and models for measuring mental concentration. Finally, the relations between the various models will be discussed.

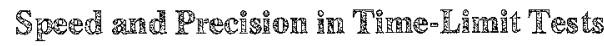

Considering the wide variety of tests used today, this paper will be restricted to a review of research on time-limit intelligence tests. Usually, these tests are found in multiple aptitude test batteries, such as the Chicago Tests of Primary Mental Abilities, the Differential Aptitude Tests, and the General Aptitude Test Battery (GATB).

Traditionally, a distinction is made between two types of mental tests, viz., speed and power tests (see, e.g., Lord \& Novick, 1968). Suppose a random variable I, a person's total incorrect score, is defined as a sum of two random variables $U$ and $W$, the number of items not attempted (U), and the number of items to which an incorrect answer was given (W). That is, $I=U+W$. Now, a pure speed test is defined as a test for which the random variable $W$ has a degenerate probability distribution, such that $P(W=0)=1$. A pure power test is defined as a test that is administered in such a way that $P(U=0)=1$. Hence, pure power tests satisfy the relation $I=W$ and pure speed tests satisfy the relation $I=U$. Time-limit tests can be seen as a combination of a pure speed test and a pure power test. As such, this definition should have an impact upon the test scores.

When a time-limit test is administered under a liberal time condition, i.e., as a power test, and, in addition, the testee is urged not to guess but to choose the do not know alternative, there should remain only two response categories: correct and do not know. Some case studies using a time-limit intelligence test designed for 11 - to 12 -year-old children, administered to 5- to 6-year-old children, support this assertion. Items that are answered with do not know either appeal to factual knowledge that the testee does not (yet) possess (e.g., the meaning of an unfamiliar word) or appeal to algorithmis or a specific problem-solving strategy that is not (yet) known to the individual (e.g., solving a set of linear equations or the simultaneous rotation of two three-dimensional objects). Hence, variation in test scores has to be attributed to variation in speed, assuming the do not know category is absent. This assumption applies to most time-limit intelligence tests, certainly for the tests referred to in this paper.

Time-limit tests typically use a restricted time condition, i.e., no one will solve all itens. The restriction in time is conveyed to the testee in the instruction, e.g., Work as fast as you can, but try not to make mistakes? In addition, time-limit tests typically consist of a set of items with at least one correct alternative and at least one false alternative (viz., multiple-choice items). Hence, all time-limit tests are characterized by the following points:

1. They are administered with a time limit.

2. They use a speed-accuracy instruction.

3. For each item an incorrect response can be given.

In scoring time-limit tests, five response categories are likely to be used:

1. The number of items correct (correct score); 
2. The number of items incorrect (incorrect score);

3. The number of invalid answers, i.e., the response was not of the correct format;

4. The number of items skipped, i.e., the item may have been attempted, but no answer was recorded; and

5. The number of items attempted (attempted score).

In most tests the frequencies in Categories 3 and 4 are negligible.

Ignoring invalid and skipped items, the correct score may be written as $r=a-f$, where $r$ denotes the number of items correct, $a$ the number of items attempted, and $f$ the number of items incorrect. Usually, the number-correct score is used as the basic score. It is easily seen that mere attention to the number of items correct without regard to the number of items attempted can be misleading. The question arises as to how the number-correct score is to be interpreted in relation to the number of items attempted. Also, the meaning of the attempted score as such needs clarification.

To answer these questions the authors initially developed models that could account for the observed data, for example, a frequency distribution of the test score or a persons-by-items matrix of one-zero, or correct-incorrect, scores. Two models for the number-correct score in relation to the number-attempted score-the binomial error model and the Rasch model-and a model for the attempted score, the Poisson-Erlang model, will be discussed.

\section{The Binoringial Error Model}

The two models that will be discussed in this and the following sections have originally been developed for power tests, i.e., task-limit tests, that is, tests in which all testees have attempted all items. Therefore, these models cannot be applied to time-limit tests without special provisions. In order to make application possible, the total set of persons is partitioned into classes according to the number of items attempted. For each class the test may then be regarded as a task-limit test consisting of $a$ items (capital letters will be used for random variables and lower case letters for the specific values they take). Within each class of persons the frequency distribution of the number of items correct may be observed. Van der Ven (1969) has proposed the binomial error model (see also Lord \& Novick, 1968) as a possible model that reproduces this frequency distribution.

The binomial error model implies that the probability of a correct response, given that a person has attempted a specified number of items, depends only upon the person. Thus, given a particular person, all items attempted have an equal probability of being answered correctly. This assumption is called the constancy hypothesis (van der Ven, 1969, p. 5). The idea underlying the constancy hypothesis is that if an item increases in difficulty, the person spends more time on it, choosing his or her response time such that his or her probability of a correct response remains constant. Thus, it is quite possible that the constancy hypothesis applies, even if the items increase in difficulty, provided the person spends more time on the more difficult items.

A statistical test of the model. The constancy hypothesis implies that for a given person characterized by a particular number of items attempted, the number of items correct follows a binomial distribution with correct-response-probability $\pi$ which is person dependent, that is,

$$
P\left(R_{i}=r \mid A_{i}=a\right)=\left(\begin{array}{l}
a \\
r
\end{array}\right) \pi_{i}^{r}\left(1-\pi_{i}\right)^{a-r} .
$$

where a person is indicated by $i$, and capital letters are used for random variables.

Assuming a probability distribution for $\pi$ (for example, a beta distribution, often called the natural prior distribution for $\pi$ ), the distribution of the number of items correct over persons can easily be obtained. However, there is little substantial evidence for choosing a beta distribution as the mixing 
distribution. In fact, mathematical convenience is the main reason for the popularity of the beta distribution.

Given these parameters, the distribution of the number of items correct can be computed and compared with the observed frequencies. Thus, the fit of the model, and an evaluation of the choice of the beta distribution, can be assessed through some sort of goodness-of-fit test, e.g., Pearson's chisquare test or the Kolmogorov-Smimov test (see, e.g., Mood, Graybill \& Boes, 1974).

The model has been tested (van der $V e n, 1969$ ) on the GATB, and on the intelligence part of a Dutch test battery for Occupational Interest, School Achievement, and Intelligence (ISI; Snijders, Souren, \& Welten, 1963; Snijders \& Welten, 1968). The GATB consists of 12 tests (8 paper-and-pencil and 4 apparatus tests) that have been selected because they are good measures of nine occupational aptitudes. The model was tested on the first seven verbal subtests. Data were not available on the performance subtests ( 4 apparatus, 1 paper-and-pencil). The model gave a reasonably good account of the data, especially, for the tests Name Comparison, Computation, Tool Matching, and Form Matching. For Arithmetic Reasoning the fit was less impressive. Also for the Three-Dimensional Space and Vocabulary tests the results were questionable. For the ISI tests, the binomial error model showed a significant lack of fit.

Precision using the binomial error model. If the binomial error model holds, it can be shown that the proportion of the number of items correct (relative to the number of items attempted) is an un. biased estimator of the correct-response-probability, i.e., precision. This provides a comparison of different people with different numbers attempted. Two individuals with scores $r=18$ and $a=20$, and $r=27$ and $a=30$, respectively, have the same proportion correct, namely .90. As to precision, there is a problem related to the scale of measurement. As a probability, it runs from zero to one, whereas it is eventually desirable to have a score on the real axis. A possible transformation could take the following form:

$$
\xi_{i}=\sigma+\ln \frac{\pi_{i}}{1-\pi_{i}} \text {. }
$$

where $\sigma$ is an arbitrary constant related to item difficulty and In is the natural logarithm. This formula is a special case of the Rasch model, which will be discussed in the next section. In this paper $\xi$ will be called precision, or accuracy.

This definition of precision is close to Spearman's goodness-of-response or clearness concept:

Does our $g$ - measured as it is by mental tests-involve 'power,' in the sense of goodness of response under most favourable conditions? Or, as some writers are asserting, does it only involve speed? To answer this question in respect of any kind of ability, the first natural step is to obtain a measure of it under such conditions that the influence of speed is somehow eliminated. For this purpose, we cannot simply take the number of right responses given by the testee; for here speed is obviously influential. But there is less objection to taking instead, the proportion of right responses to the total number. (Spearman, 1927, p. 247)

The most obvious explanation for the lack of fit in case of the ISI tests is that the constancy hypothesis has been violated in these tests. An indication for this violation is given by the correct-response probabilities for different items and different subtests. In Table 1 the proportions of individuals correctly responding to the first 11 items of the $6 \mathbb{I S I}$ tests are shown as reported by Van den Wollenberg (1979). Van der Ven (1969) reports similar results.

The differences between the proportions clearly indicate that the constancy hypothesis has to be rejected. The rejection of this hypothesis leads to rejection of the proportion of items correct as an unbiased estimate of precision. Hence, the appropriateness of the correct-response-probability as a measure for precision must be questioned. 


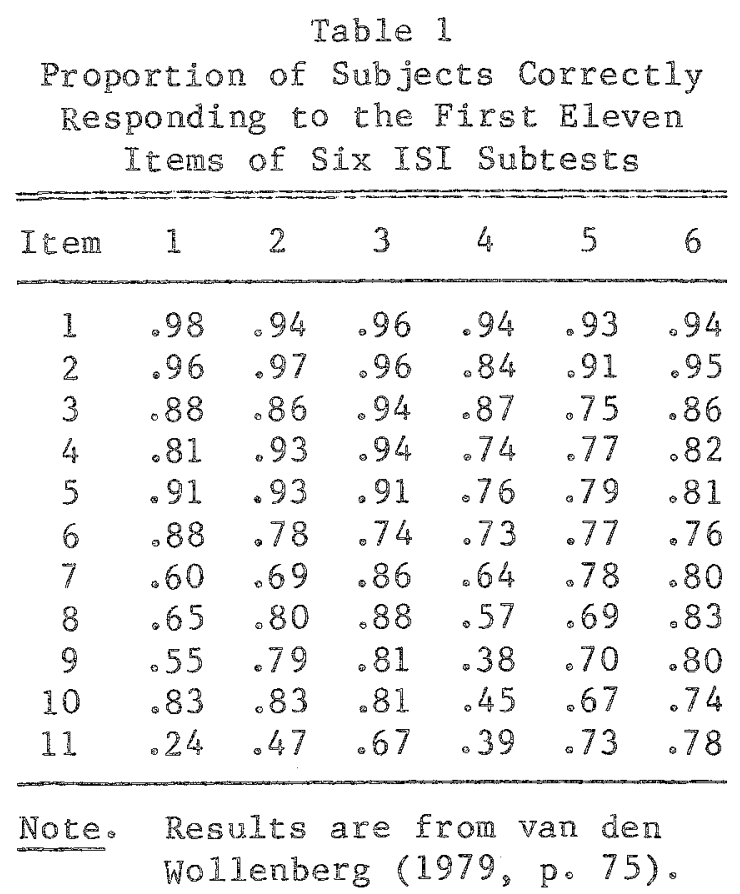

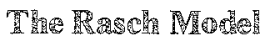

An evaluation of the fit of the binomial error model using ISI data yielded significant deviations in all cases. Consequently, the model had to be rejected for those tests. As has been seen, the most obvious explanation for the lack of fit in case of the ISI tests is that the constancy hypothesis has been violated. In such a case the correct-response-probability is not only determined by a person parameter, say $\xi$, but also by an item parameter, say $\sigma$. The relation between these parameters and the number of items correct is given by the item characteristic function $f(\xi, \sigma)$. A model that uses item characteristic functions is a member of the class of latent trait models, which is in turn a member of the class of latent structure models (see, e.g., Lazarsfeld \& Henri, 1968; Van den Wollenberg, 1979). In all latent structure models the relation between maniest and latent variables is given by

$$
f\left(\underline{X}_{i j}=\underline{x}_{i j} \mid j, \xi_{i}\right)=f_{j}\left(\xi_{i}\right) \text {. }
$$

The probability that a manifest stochastic variable $\mathbb{X}$, of person $i$ and item $j$, takes the value $x$ is given by an item characteristic function which is determined by item $j$ and the latent person parameter $\xi_{i}$ of that individual. The various latent structure models are generated by specifying the function $f_{j}$. Andersen and Madsen (1977) and Andersen (1980) have generalized this model in such a way that available information with respect to the population distribution of the person parameter can be used.

Using the logistic function, a latent trait model, which can be shown to have some very elegant mathematical and methodological properties, can be obtained-the Rasch (1960) model. Here the item characteristic function is defined as

$$
\pi_{i j}=f\left(\xi_{i}, \sigma_{j}\right)=\frac{\exp \left(\xi_{i}-\sigma_{j}\right)}{1+\exp \left(\xi_{i}-\sigma_{j}\right)} \text {. }
$$


where $\xi_{i}$ will be called the precision of person $i$ and $\sigma_{j}$ the difficulty (in the sense that a difficult item is an item that easily evokes an incorrect answer) of item $j$.

Using the following set of axioms, the Rasch model can be deduced instead of postulated (see, e.g., Fischer, 1974):

1. Unidimensionality, i.e., responses depend upon only one underlying continuum of items and persons.

2. Monotonicity, i.e., all item characteristic functions are strictly monotone in the latent trait.

3. Local stochastic independence applies.

4. Sufficiency of simple sum statistics applies, i.e., the Fisher-Neyman factorization theorem for the likelihood function applies for the simple sum (see, e.g., Mood et al., 1974. For an in-depth discussion of sufficient statistics in latent trait models, the reader is referred to Andersen, 1977.)

5. The test items are dichotomous.

It follows (Rasch, 1961, 1966) that in the class of probabilistic unidimensional models with dichotomous manifest variables, the Rasch model implies a very mice methodological property, i.e., specific objectivity. In fact, in this class of models, the Rasch model is necessary and sufficient for specific objectivity. Specific objectivity implies that the outcome of a comparison of persons does not depend on the specific items used, provided that the items belong to the same dimension. Hence, the measurement of the latent trait is not dependent upon the selection of the items. As this is the aim of all studies that use measurement devices for latent traits, it appears that the Rasch model is one valid device to do so.

A statistical test of the model. Using standard methods, it is easily shown that a sufficient statistic for the latent person parameter $\xi_{i}$ is given by

$$
S_{i}=\sum_{j=1}^{k} x_{i j}
$$

where $X_{i j}$ is the score of person $i$ on item $j$. Furthermore, it can be shown that the joint probability of response vectors of all persons conditional upon their total test score, written as

$$
L_{c}=\prod_{i=1}^{n} P\left(X_{i 1}=x_{i 1}, \ldots, X_{i k}=x_{i k} \mid s_{i}=s_{i}\right) .
$$

is independent of the person parameter $\xi_{i}$. This function, called the conditional likelihood function, is used to obtain estimates for the item parameters (Andersen, 1973). It is important to note that these estimators are independent of the person parameters. If conditionality is dropped, the unconditional joint probability function of all response vectors is given by

$$
L=\prod_{i=1}^{n} P\left(x_{i 1}=x_{i 1}, \ldots, x_{i k}=x_{i k}\right) \text {. }
$$

Andersen (1973) has shown that this function is composed of two independent components, i.e., the unconditional likelihood can be written as

$$
\mathrm{L}=\mathrm{L}_{\mathrm{c}} \because \mathrm{L}_{\mathrm{m}}
$$

where $L_{c}$ is given by Equation 6 and $L_{m}$ is a function that is determined by the person parameters and the item parameters. Substituting the item parameter estimates obtained from Equation 6 , estimates of the person parameters are obtained by maximizing $L_{m}$ with respect to these parameters. 
Given these parameters, test scores can be predicted and the fit of the model can be evaluated by comparing observed and predicted scores. Several such tests have been developed. More recently, Van den Wollenberg (1979) has proposed two tests, one of which is especially sensitive to violations of Axiom 1 (unidimensionality), besides the violations detected by other tests, e.g., the likelihood ratio test of Andersen. Moreover, Van den Wollenberg (1979) shows that the usual tests are insensitive to violations of unidimensionality.

The six intelligence subtests of the ISI have been tested by Van den Wollenberg (1979) for Rasch homogeneity. (This expression is used when the model holds.) Except for a few items, the Rasch model did hold for the first five tests, but not for the sixth-Figure Categories. Further analysis of this test showed that the items appeared to be very heterogeneous, both with respect to item discriminations and dimensionality. It follows that the test is not adequate and reconstruction seems necessary.

Precision using the Rasch model. If the Rasch model holds for a particular test, any subset of its items can be used to measure $\xi$ in a specifically objective way. For a person who has attempted 11 items of a Rasch homogeneous test, these 11 items can be used to estimate $\xi$, precision. (The estimated value of $\xi$ is denoted by $X$ taking the specific value $x_{0}$ ) For a person who has attempted all items, the complete test can be used. The parameter estimates for all persons with a given number of attempted items can be obtained by means of a method described by Fischer (1974, p. 215). Transformations (see Van den Wollenberg, 1979, p. 180) can be obtained such that persons with different numbers of items attempted may be measured on the same scale. For example, for ISI Test 4, Rotation, the $x$-value for a person with $r=8$ and $a=1$ is equal to .82 , while the $x$-value for a person with $r=10$ and $a=20$ is equal to 1.27. Their $\hat{\pi}$-values would be the same, but it is known that the binomial error model does not hold. Precision still can be measured independently from number of items attempted, provided that the Rasch model holds independently of a person's speed (as expressed by the number of items attempted).

\section{Suramemparay}

Using the Rasch model, Van den Wollenberg (1979) has shown that for the ISI tests the binomial error model had to be rejected due to violation of the constancy hypothesis introduced by van der Ven (1969). Application of the Rasch model on data of the GATB and other time-limit intelligence tests is the object of present and future research.

Rejection of the binomial error model implies the rejection of the proportion of the number of items correct as a measure for precision. Van den Wollenberg (1979) showed that provided the Rasch model holds, the latent person parameter of the Rasch model can be used as an estimator of precision. Given the methodological properties of the Rasch model, there is a specific objective measure of precision.

\section{A Model for the Nuringer of}

The mathematical expectation of the number of items correct for a specific person, given a specified number of items attempted, can be obtained from his or her precision. The unconditional num. ber of items correct is also dependent upon the number of items attempted, however. The question arises as to the nature of the attempted score.

If there is interest in developing a model for the number of items attempted, it should be taken into account that the number of items attempted is dependent upon the time a person needs for each individual item - the shorter these durations, the larger his/her number attempted. Hence, before de- 
signing a model for the number of items attempted, it is necessary first to develop a model for the time a person needs for an individual item. This time will be called response time. Such a model has been developed by van der Ven and Pieters (1977).

The model proposed is based on a theoretical framework which describes the underlying operational characteristics of a problem-solving person. Although the framework is rather general, this paper will be confined to tasks for which the steps that have to be taken to arrive at the solution, called the algorithm, are known to the individual.

The basic idea of the model is that the solution of a problem requires a serial process that involves solving a number of subproblems. Following McClelland (1979) the model will be called a discrete process model. Assuming that the algorithm used by the person is known, it is possible to construct a finite machine that performs such a task without mistakes. Given the working speed of that particular machine, the total response time is constant.

It is clear, however, that human performance is not merely determined by working speed. From time to time attention fades away, the solving strategy may vary due to learning effects, and performance may be influenced by such effects as latigue. For the moment, assume that learning effects and fatigue are absent. For reasons of simplicity, then, it is assumed that, while executing a task, a person can be in one of two states: processing and nonprocessing. The term nonprocessing is used to denote all processes that are not directly related to or necessary for solving the problem. In the following the concept distraction will be used to describe these processes. Hence, the total response time or response latency (reaction time) is composed of two components - the total processing time, l.e., the sum of response times for the different operations or steps in the algorithm, and the total distraction time, i.e., the total time spent in the state of distraction.

The process described above resembles a two-state Markov model in continuous time. Now suppose the person is confronted with a number of similar iterns, i.e., items with equal difficulty level. Then, the total time spent in the state of processing is fixed over items, provided the algorithm is known to the person. Hence, if the general psychological state of the individual is constant (viz., no learning effects, no fatigue effects, and no variation in motivation, the variation in the total processing time will be negligible compared with the variation in the total distraction time. Hence, small fluctuations in the processing times are neglected. These fluctuations, however, may become important in fast, simple reaction time tasks. For all practical purposes, thetn, the total processing time is assumed fixed.

Introduction of some technical assumptions and specification of the procedure that is used in col. lecting the response data (e.g., response times or number of responses within a fixed time) leads to a specific model.

\section{The Poำs}

In this section the distribution function for the total response time will be derived, asing the theoretical framework that was outlined above. In doing so, some elementary mathematical resulis in calculus and probability theory will be used.

The basic idea of the theoretical framework was that the total response time $(T)$ is composed of two independent components - the total processing time denoted by the symbol $a$ and the total distraction time denoted by the symbol $\Delta$, which was defined as a sum of distraction times $\left(\Delta_{l}\right)$. The formwla is

$$
T=\alpha+\Delta .
$$

Downloaded from the Digital Conservancy at the University of Minnesota, http://purl.umn.edu/93227.

May be reproduced with no cost by students and faculty for academic use. Non-academic reproduction requires payment of royalties through the Copyright Clearance Center, http://www.copyright.com/ 
As was pointed out before, it is plausible to assume that the total processing time $(\alpha)$ is fixed. This implies that the distribution of the total response time is determined by the distribution of the total distraction time, $\Delta$.

Concerning the total distraction time, two technical assumptions are made. The first assumption is that each distraction time $\Delta_{l}, 1=1,2, \ldots, k$ has an exponential distribution with parameter $\delta$, such that

$$
f_{\Delta_{1}}(t)=\delta e^{-\delta t} \quad \text { for } \delta>0, t>0 \text {. }
$$

Using standard results from probability theory, it is easily shown that the sum of the $k$ independent exponentially distributed random variables representing distraction times (i.e., the total distraction time, $\Delta$ ) has an Erlang distribution, which can be written as

$$
\mathrm{f}_{\Delta_{\mathrm{k}}}(t)=\frac{(\delta t)^{(k-1)} \delta \mathrm{e}^{-\delta t}}{(k-1) !} \text { for } t>0 \text { and } \delta>0 \text {. }
$$

The total distraction time is defined as the total time spent in the state of distraction. Given this interpretation, it is plausible to assume that the number of entries in this state is not fixed but is itself a random variable.

The second assumption is that at any time during processing, a distraction may occur. Assuming independence of these events and assuming that the probability of such an event is independent of the time of occurrence, the number of distraction periods $K$ has a Poisson distribution with parameter $\gamma$ :

$$
P(K=k)=\frac{\gamma^{k} e^{-\gamma}}{k !}, \quad k=0,1,2, \ldots .
$$

From the conditional cumalative distribution function of the total distraction time $F\left(\Delta_{k} \mid K=k\right)$, the unconditional distribution function $F(\Delta)$ can be obtained by taking the expectation of $F\left(\Delta_{k} \mid K=k\right)$ with respect to $K$. The result can be written as

$$
\begin{aligned}
\mathbb{F}_{\Delta}(t) & =P(\Delta<t \mid K=0) P(K=0)+\sum_{k=1}^{\infty} P(\Delta<t \mid K=k) \cdot P(K=k) \\
& =e^{-\gamma}+e^{-\gamma} \sum_{k=0}^{\infty} \frac{\gamma^{k+1}}{k_{0}^{!}(k+1) !} \Gamma_{\delta t}(k+1) .
\end{aligned}
$$

where

$$
\Gamma_{\delta t}(k+1)=\int_{0}^{\delta t} e^{-x j} x d x
$$

denotes the incomplete gamma function. Since $k$ is discrete,

$$
\mathbb{F}_{\Delta}(t)=1-e^{-(\gamma+\delta t)} \sum_{k=0}^{\infty} \frac{\gamma^{k+1}}{(k+1) !} \sum_{j=0}^{k} \frac{(\delta t)^{j}}{j !} \text {. }
$$

may be written. From Equation 9 it can be seen that the distribution function of the total response time $T$ is defined by the distribution of the total distraction time, $\Delta$, except for a change of the origin. Using the simple transformation $T=\alpha+\Delta$ and thus substitution of $(t-\alpha)$ for $t$ in Equation 15 gives 
the distribution function for $T$. A more detailed derivation and analysis of the Poisson-Erlang distribution and a comparison with other distribution functions can be found in Pieters (1981).

The moments of this distribution are easily obtained, using the well-known formula

$$
E\left(t^{r}\right)=E_{K}\left[E\left(t^{r} \mid K=k\right)\right]
$$

The expectation of $T, E(T)$, may be written as

$$
E(T)=\alpha+\frac{\gamma}{\delta}
$$

its variance, $\sigma^{2}(T)$, as

$$
\sigma^{2}(\mathrm{~T})=2 \frac{\gamma}{\delta^{2}}
$$

and its third central moment, $\mu_{3}(T)$, as

$$
\mu_{3}(T)=6 \frac{\gamma}{\delta^{3}} \text {. }
$$

\section{Estimmtion of the Paratineters}

In order to test the Poisson-Erlang model, appropriate estimators for the parameters have to be computed. In the statistical literature a great variety of estimation procedures can be found (see, e.g., Kendall \& Stuart, 1967). However, the most widely used methods are the method of moments and maximum likelihood estimation.

Maximum likelihood estimators are defined as follows. Let $X$ be a random variable with probability density function $f_{X}$ depending on the parameters $\tau_{1}, \ldots, \tau_{k}$. Suppose there is a random sample consisting of the independent random variables $X_{1}, \ldots, X_{n}$, each with distribution function $f_{X}$ then the joint probability density function $f_{X}\left(X_{1}=x_{1}, \ldots, X_{n}=x_{n} \mid \tau_{1}, \ldots, \tau_{k}\right)$ can be written as

$$
\prod_{i=1}^{n} f_{X}\left(X_{i}=x_{i} \mid \tau_{1}, \ldots, \tau_{k}\right) \text {. }
$$

Maximum likelihood estimators $\widetilde{\tau}_{1}, \ldots, \tilde{\tau}_{k}$ of $\tau_{1}, \ldots, \tau_{k}$ are found by maximizing Equation 20 with respect to these parameters. ${ }^{1}$

Although maximum likelihood estimators have some useful statistical properties, method-of-moments estimators are widely used because of the ease in computation. The latter class is defined as follows: Suppose there is a random variable $X$ associated with a $k$-parameter distribution. Generally, the moments of this distribution are simple functions of these parameters. Choosing a set of $k$ moments, replacing population moments by sample moments, and solving the resulting $k$ equations for the $k$ parameters leads to method-of-moments estimators. In the case of the Poisson-Erlang model the moment equations (Equations 17 through 19) must be solved for the parameters $\alpha, \delta$, and $\gamma$. The result can be expressed as

$$
\hat{\alpha}=m_{1}^{3}-\frac{9 m_{2}^{2}}{6 m_{3}} .
$$

${ }^{1}$ Derivation of maximum likelihood estimators for the Poisson-Erlang model would go beyond the scope of this paper. The interested reader is referred to Pieters (1981). 


$$
\begin{aligned}
& \hat{\gamma}=\frac{9 m_{2}^{3}}{2 m_{3}^{2}} \\
& \hat{\delta}=3 \frac{m_{2}}{m_{3}}
\end{aligned}
$$

where $m_{1}^{\prime}$ denotes the sample mean, $m_{2}$ the sample variance, and $m_{3}$ the sample third central moment. Monte carlo studies have indicated that these estimators have a number of disadvantages (viz., the variance is large; due to sampling fluctuation the moment estimator for $a$ can become greater than the mean of $T$, which is absurd). In order to avoid these complications, a modified procedure was used, which is based on the process model previously outlined.

Suppose $\alpha$ is known, modified method-of-moments estimators for $\delta$ and $y$ are given by

$$
\hat{\delta}=\frac{2\left(m_{1}-\alpha\right)}{m_{2}}
$$

and

$$
\hat{\gamma}=\frac{2\left(m_{1}-\alpha\right)^{2}}{m_{2}}
$$

From the process model it can be concluded that a must have a value that is close to the smallest observed latency. The procedure used to arrive at an estimator for $a$ is as follows. Choose a starting value for $\alpha, e . g$. the smallest observed latency minus some small constant, compute modified moment estimators for $\delta$ and $\gamma$ according to the formulas given above, and evaluate the fit using a goodness-offit test, e.g., the Kolmogorov-Smimov test. Then choose a new value for $\alpha$ and repeat the procedure until the statistic stabilizes. In fact, a minimum distance estimator for $\alpha$ (Mood et al., 1974) is used in combination with method-of-moments estimators for $\delta$ and $\gamma$. Although the properties of these estimators have not yet been studied in detail, application gives at least insight into the fit of the model.

Earlier in this paper it was argued that human performance is not merely determined by working speed, that is, it was assumed that learning effects and fatigue are absent. In terms of the parameters of the Poisson-Erlang model, this implies that there are no trend effects in the parameters. From the moment equations it is clear that a trend in processing time will result in a trend in the mean. Trend in distraction parameters will result in trend effects in mean and variance. Hence, the assumptions made in construction of the model are easily varified by inspecting the mean and variance for trend. In addition to long term trend effects, the data have to be inspected for cyclical trend effects in the parameters. These effects manifest themselves in the same way as ordinary long term trend effects.

Recapitulating, it can be said that before testing the model, the actual time series has to be investigated for possible long term trend effects, in order to check whether the person has worked with the same speed during the test. Habituation or fatigue effects or a change in motivational attitude, such as the willingness to work fast, can play a role. Long term trend effects, which are considered to be the result of a gradual change in speed, must be eliminated before the model is tested. Also, a check on short-term periodicity has to be made. 


\section{A}

The Poisson-Erlang model has been developed based on the process model outlined. Because of the strong connection between this process model and the resulting stochastic model, there are a number of restrictions as to the applicability of the model to real test data.

First, there is a restriction on the difficulty of the items in the test. It is assumed that the processing time is constant, reflecting the fact that the number of operations is constant. This means that the items may not differ in difficulty. Varying levels of difficulty of the items may affect parameter $\alpha$ but also parameter $\gamma$. From the process model, is is clear that an increase in difficulty level will lead to an increase in the total processing time, assuming all other conditions to remain equal. An increase in the number of operations, however, could also lead to an increase in the number of distractions, as the person may enter the state of distraction after each operation. In addition to these effects, the person may choose some sort of trade-of between the parameters, which may differ for the various items.

Secondly, it was assumed that the solution process is completely known to the individual. Therefore, tests wherein the solution process contains inevitable stochastic components (viz., the Raven test) such as detection or learning of the correct solving strategy are excluded.

Last but not least, all stochastic components are interpreted as distraction. Therefore, stochastic components that are not directly connected to performing the task have to be eliminated (viz., experimental procedures that introduce stochastic components in the reaction process). All stochastic components are assumed to reflect the stochastic components of the model. Hence, artifacts representing pseudo-random variation caused by experimentation must be considered. Suppose a set of words are presented to an individual - either a set with one serse word and the other non-sense words resembling sense words or a set of all non-sense words. The person is asked whether the set contains a senseword and is instructed to react as quickly and accurately as possible. Suppose all items, i.e., strings of words, are equally difficult. Then, arandom component has been introduced that is not process related but merely introduced by the place of the sense word in the string. In addition, non-sense strings will give a longer reaction time due to the fact that a person has to scan the whole string.

Generally, these conditions are met in so-called concentration tests. An example of such a test is a Dutch concentration test, the Bourdon-Wiersma test (Boeke, 1963), which was originally proposed by Oehm (1896). In this test the dependent variable is reaction time (RT). Occasionally, the dependent variable in concentration tests is the number of items completed in a fired time (i.e., a speed test). An example of this type of test is the Pauli test (Arnold, 1975) originally proposed by Kraepelin (1902). ${ }^{2}$ In the following sections, the application of the Poisson-Erlang model to the former type of tests will be discussed.

Method. The model was tested on data obtained from the so-called Alertron, an apparatus version of the Bourdon-Wiersma test. The Alertron consists of a black display $(117 \times 117 \mathrm{~mm})$ with nine red lamps in a three-by-three design $(60 \times 60 \mathrm{~mm})$. A random configuration of three, four, or five lamps is presented to the individual, who is instructed to push the button representing the configuration in question. There are two response buttons-a four and a ron-four button. After rellease of a button, a new stimulus is displayed. Hence, the testee works under a self-paced condition. As is usual in speeded tests, the individual is instructed to work as fast but also as accurately as possible. The total number of patterns presented to the testee is 1,250 . After a series of 25 consecutive patterns, the total time is registered, yielding a series of 50 response times.

\footnotetext{
${ }^{2}$ A model for this type of data based on the process model outlined above will be published later.
} 
In addition to the Alertron data, the model was tested on the paper-and-pencil version of the Alertron, i.e., the original Bourdon-Wiersma test.

Parameters were estimated using the procedures for the Poisson-Erlang model. The KolmogorovSmimov test was used to evaluate the fit between the observed and predicted reaction time distribution.

The Alertron was administered in order to study its diagnostic possibilities. The testees were chosen more or less arbitrarily. Test protocols were obtained from five different groups of individuals: 15 were hospital patients of a rehabilitation institute in Holland ("Het Roessingh," Enschede); 4 were staff members of that institute; 6 were members of the mathematical psychology group of the University of Nijmegen; 3 were children of about 10 years old; and 7 were individuals about whom no information was available. The total number of testees from these five groups was 35 .

Results. The values of a varied from about 10 to 25 seconds, those of $\delta$ from about 1 to $10\left(\mathrm{sec}^{-1}\right)$, and those of $\gamma$ from about 10 to 25 (these figures apply to a row of 25 dot-patterns). In practically all cases the model was not rejected.

Also available were the results of 20 test administrations of a single individual over a 4 -month period. The parameter estimates of this individual showed large differences over time. The parameter estimate $\alpha$ varied from about 15 to 20 ; the parameter estimate $\delta$, from about 1 to $4 ; \gamma$, from about 10 to 20. Changes in $\delta$ seemed to be related to the general mental state of the individual.

Repeated test administrations during the same day of another individual also showed remarkable differences in parameter values, suggesting that speed and distraction (especially parameter $\delta$ ) were related to the hour of the day.

The paper-and-pencil data were used to compare the fit of the Poisson-Erlang model with the fit of alternative $\mathbb{R T}$ models. In the literature, a wide variety of probability distributions are proposed to account for $\mathrm{RT}$ data. However, few models are based on a psychologically meaningful process model that applies in the case of concentration tests. An exception has to be made for two models:

1. The gamma distribution, which was proposed by a number of authors (e.g. Bush \& Mosteller, 1955; Christie, 1952; Luce, 1960; Resthe, 1961) and is, in fact, a special case of the Poisson-Ering distribution, arising if the number of distractions is assumed to be constant.

2. A model proposed by Hohle $(1965,1967)$ and Ratcliff $(1978,1979)$, which arises as a convolution of the nomal and exponential distribution functions, where the exponential part accounts for some sort of decision process and the normal distribution arises as the sum of all other processes using the centrallimit theorem.

The parameters of the three-parameter gamma distribution were estimated using a procedure similar to the estimation procedure previously described. As the Hohle model lacks a base parameter ( $\alpha$ in the Poisson-Exlang model) the straightorward method-of-moments procedure was used. The results are shown in Table 2, which displays the rank ordering of the Kolmogorov-Smimov goodness-offit statistic for the three models. (Note that the three models contain an equal mumber of parameters.) The results clearly indicate that the Poisson-Erlang model gave a consistently better fit than the other models even though the differences were small.

Apart from a comparison of the fit of the three models, the RT data were analyzed using a method proposed by Thomas and Ross (1980). They considered diperent procedures that can be used to combine RT distributions belonging to the same lamily of densities into a new, so-called, group RT distribution belonging to the same familly. Thomas and Ross showed that the group distribution can be obtained through Vincentizing procedures. Vincentizing gmounts to averaging the quantiles of the individual $R T$ distributions. The family to which the resulting group distribution belongs depends upon the transformation on the quantiles before averaging. An indication for the correct transiorma-

Downloaded from the Digital Conservancy at the University of Minnesota, http://purl.umn.edu/93227. May be reproduced with no cost by students and faculty for academic use. Non-academic reproduction requires payment of royalties through the Copyright Clearance Center, http://www.copyright.com/ 
Table 2

Rank Ordering of the Kolmogorov-

Smirnov Goodness-of-Fit Statistics

for Three RT Models

\begin{tabular}{lccc}
\hline $\begin{array}{l}\text { Poisson } \\
\begin{array}{l}\text { Erlang } \\
\text { Mode1 }\end{array}\end{array}$ & $\begin{array}{c}\text { Hohle } \\
\text { Model }\end{array}$ & $\begin{array}{c}\text { Gamma } \\
\text { Model }\end{array}$ & Frequency \\
\hline 1 & 2 & 3 & 108 \\
1 & 3 & 2 & 1 \\
2 & 1 & 3 & 52 \\
2 & 3 & 1 & 1 \\
3 & 1 & 2 & 1 \\
1 & $*$ & 2 & 17 \\
2 & $*$ & 1 & 17 \\
\hline
\end{tabular}

* No evaluation possible due to

negative parameter estimates.

tion is given by the mean intercorrelation of the sample quantiles, given a specific transformation. Thomas and Ross (1980) suggest the following procedure:

1. Compute the quantiles $Q_{p}$, where $p=.05, .10, \ldots ., 95$ for each sample $R T$ distribution.

2. Choose a transformation $T(t)$, from the set $\left\{\log (t), t^{u}, u=-1,-1 / 2,1 / 2,1,2\right\}$.

3. Compute the average intercorrelation between the sample quantiles for each transformation.

They show that the maximum intercorrelation for the gamma distribution and the Hohle distribution is reached with the transformation $T(t)=t$. A small monte-carlo experiment using the Poisson- $\mathbb{E}$ rlang distribution performed by the present authors showed that $T(t)=t^{-1}$ is the appropriate transformation for this family.

The results of the Thomas-Ross analysis using $197 \mathrm{RT}$ samples are shown in Table 3 . The analysis indicates that the Poisson-Erlang distribution is the more appropriate model for these data although the differences are small. Moreover, the analysis sugests that none of the RT distributions given in the literature apply in this case, as all models imply the transformation $T(t)=t$.

\section{\$umamagrary}

A model was introduced that accounts for $\mathrm{RT}$ distributions in terms of two psychologically meaningful concepts-total processing time and total distraction time. Using RT data from two versions of a Dutch concentration test, the Bourdon-Wiersma test, the analysis showed that the Poisson-Erlang model gave an adequate representation of the underlying psychological process. In addition, it was shown that the fit of the proposed model was generally better than the fit of other models. As to the parameter estimation procedure, it can be concluded that this procedure gave fairly reliable estimates of the basic parameters of the model.

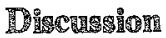

Traditionally, time limit tests are scored with the number of items correct. The studies mentioned above have shown that this score is a composite of precision, speed (total processing time), and dis- 
Table 3

Mean Intercorrelation between Sample

Quantiles after a Given Transformation

$(\mathrm{N}=197)$

\begin{tabular}{cc}
\hline Transformation & $\begin{array}{c}\text { Mean } \\
\text { Intercorrelation }\end{array}$ \\
\hline $\log _{e}(t)$ & .9827 \\
$t^{-1}$ & .9991 \\
$t^{-\frac{1}{2}}$ & .9731 \\
$t$ & .9831 \\
$t^{\frac{1}{2}}$ & .9931 \\
$t^{2}$ & .9821 \\
\hline
\end{tabular}

traction (total distraction time). From the number of items correct alone, no conclusions can be derived about the relative contribution of each of these factors. The proportion of items answered correctly relative to the number of items attempted is a very rough estimation of precision, and certainly biased in the case where the binomial error model does not hold. The number of items attempted is in fact a composite of speed and distraction. Thus, it seems necessary to develop new tests and scoring methods that enable the attainment of independent estimates of the various components. Tests that yield a time or performance series look promising.

It is very plausible that speed and precision are related within a person. For example, Spearman has already argued that:

any increase in speed at a mental operation tends towards a decrease in its goodness, whilst in. versely a greater goodness can always be attained by some sacrifice of speed. Apart from more or less accidental further influences, then, high accuracy and low speed have a perfect (though inverse) correlation. ( Spearman, 1927, p. 250)

Van den Wollenberg (1979) arrived at the same conclusion analyzing ISI tests with the Rasch model. Now, if speed and precision are related in some way, then they are both manifestations of a more fundamental ability. It seems better to measure this ability itself than its variable manifestations. In order to do so, however, it seems necessary to administer the test several times with different speedaccuracy instructions. In order to measure the underlying ability, more has to be known about the speed-accuracy trade-off function. Another problem is related to the way the instruction is taken by the testee. It could very well be that an instruction stressing speed will lead to a decrease in distraction and no change in processing speed, as the number of items attempted can also be increased by decreasing distraction. More research must be done in order to answer these problems.

Although in the studies mentioned above, the number of items correct is considered as a composite of more fundamental factors such as speed, precision, and distraction, it would be a fallacy to consider them as factors in a factor analytic sense. The approach shown in the above studies is not an alternative to the factor analytic approach leading to so-called factor theories such as Spearman's $(1904,1927)$ two-factor theory, Thurstone's (1938) multiple-factor theory, and Guilford's (1967) structure-of-intellect model. The factor analytic studies that have led to these theories are all based on tests that have been scored with the number of items answered correctly. The question is whether it is ap- 
propriate to apply factor analysis on such a raw score, which has no justification in a theory of mental measurement.

The meaning of the studies mentioned above lies precisely in the fact that they question the use of number correct as the dependent variable in further analyses. This has led to the development of theories and models that entail measurement as a natural result. Number correct as a test score has been reduced to two more fundamental quantities: the ability underlying speed and precision, and distraction. Tentatively, it may be concluded then that factor analytic studies, if any, should be based on these variables, as they are psychologically meaningful instead of on number correct, which has no explicit justification.

Another argument for separating ability and distraction in factor analytic studies is that ability as a personality construct is probably task specific, while distraction would not be task specific but may depend on the demands of the task and extraneous conditions like motivation. Ability might be defined as a person's precision or response accuracy per unit processing time. If only number correct (which contains distraction) is used in factor analysis, the outcome could be less definite and possibly misleading.

The present line of research, more specifically model-oriented research, enables the introduction of theoretically defined constructs. For example, within the Poisson-Erlang model a personality trait called concentration could be defined as a person's distraction time per unit processing time. Hence, from a vague concept, concentration has become a theoretically defined construct. These definitions, however, are based on the interpretation of the three model parameters. In order to strengthen the base for this interpretation, the first author is currently investigating the way in which task variables affect the parameters in the Poisson-Erlang model.

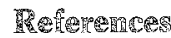

Andersen, $E$. B. Conditional inference and models for measurizg. Koperhagen: Mental Hygiejnisk Forsknings Institut, 1973.

Andersen, E. B. Sufficient statistics and latent trait models. Psychometrika, 1977, 42,69-81.

Andersen, $\mathrm{E}$. B. Comparing latent distributions. Psychometrika, 1980, 45,121-134.

Andersen, E. B⿺辶, Madsen, M. Estimating the parameters of the latent population distribution. Psychometrika, 1977, 42,357-374.

Arnold, W. Der Pauli test. Berlin: Springer, 1975.

Boeke, $\mathbb{P}$. E. Psychodiagnostische problemen van de epilepsie. Assen, The Netherlands: van Gorcum, 1963.

Bush, R. R., \& Mosteller, F. Stochastic models for learning. New York: Wiley, 1955.

Christie, L. S. The measurement of discriminative behavior. Psychological Review, 1952, 62, 43-50.

Fischer, G.H. Einfuhrung its die theorie psychologischer tests. Bern, Switzerland: Huber, 1974.

Furneaux, W. D. Intellectual abilities and problemsolving. In H. J. Eysenck (Ed.), HAndbook of $a b$ normal psychology. New York: Basic Books, 1961.

Guilford, $\mathbb{D}$. P. Personality. New York: McGraw-Hill, 1959.
Guiford, J. P. The nature of humanintelligence. New York: MaGraw-Hill, 1967.

Hohle, R. H. Inferred components of reaction time as functions of foreperiod duration. Joutral of Experimental Psychology, 1965, 69, 382-386.

Hohle, R. H. Component process latencies in reaction time of children and adults. In $\mathbb{L}$. P. Lipsint \& Ch.C. Spiker (Eds.), Advances in child development and behavion (Vol.3). INew York: Academic Press, 1967.

Kendall, M. G., \& Stuart, A. The advanced theory of statistics ( $\forall$ ol. 2, 2nd ed.). New York: HoughtorMifflin, 1967.

Kraepelin, E. Die arbeitscurve. Wundts Psychologische Studien, 19, 1902.

Lazarsfeld, P. F., \& Henri, N. W. Latent structure analysis. New York: Houghton-Mifflin, 1968.

Lord, F.M., \& Novick, M. K. Statistical theories of mental test scores. Reading MA: Addison-Wesley, 1968

Luce, R. D. Response latencies and probability. In K. J. Arrow, S. Karlin, \& P. Suppes (Eds.), Mathematical methods in the social sciences, 1951 . Stanford: Stanford Universivy Press, 1960. 
Mood, A. M., Graybill, F. A., \& Boes, D. C. Introduction to the theory of statistics. New York: McGraw-Hill, 1974.

McClelland, J. L. On the time relations of mental processes: An examination of processes in cascade. Psychological Review, 1979, 86, 287-330.

Dehrn, A. Experimentelle Studien zur Individualpsychologie. Psychologische Arbeiten von Kraepelin. $1896,1,92-151$.

Pieters, J. P. M. The Poisson-Erlang process: A characterization and parameter estimation procedures (Technical Report 81MA01). Nijmegen, The Netherlands: University of Nijmegen, Department of Mathematical Psychology, 1981.

Rasch, G. Probabilistic models for some intelligence and attainment tests. Kopenhagen: Rielson \& Lydiche, 1960.

Rasch, G. On general laws and the meaning of measurement in psychology. In Berkeley Symposium on Mathematical Statistics and Probability. Berkeley: University of Califiomia Press, 1961.

Rasch, G. An informal report on a theory of objectivity in comparisons. In L. J. Th. van der $K^{2}$ amp \& C. A. J. Vlek (Eds.), Psychological measurement theory. Proceedings of the NUFFIC International Summer Session of Science at Het Oude Hof. The Hague, July 1966.

Ratcliff, R. A theory of memory retrieval. Psychological Review, $1978,85,59-108$.

Ratclif, $\mathbb{R}$. Group reaction time distributions and an analysis of distribution statistics. Psychological Bulletin, 1979, 86, 59-108.

Restle, F. Psychology of judgement and choice. New York: Wiley, 1961.

Snijders, J. T., Souren, C. J.M. H., \& Weiten, V.J. IST-publicaties I. Algemene handleiding. Gronin. gen: Wolters, 1963.

Snijders, J. T., \& Welten, V.J.De ISI-schoolvorderingen en Intelligentietest, vorm $I$ en $I$. Groningen: Wolters-Noordhoff, 1968.

Spearman, C. 'General intelligence,' objectively determined and measured. American Journal of Psychology, 1904, 15, 201-293.
Spearman, C. The ability of man. London: MacmilIan, 1927.

Thomas, E. A. C., \& Ross, B. H. On appropriate procedures for combining probability distributions within the same family. Journal of Mathematical Psychology, 1980, 21, 136-152.

Thurstone, L. L. Ability, motivation and speed. Psychometrika, 1937, 2, 249-254.

Thurstone, $\mathbb{L}$. $\mathrm{L}$. Primary mental abilivies. Chicago: University of Chicago Press, 1938.

Van der Ven, A. H. G.S. The binomial error model applied to time-limit tests. Nijmegen, The Netherlands: Schippers, 1969.

Van der Ven, A. H. G. S., \& Pieters, J.P. M. The Poisson-Erlang model: A probabilistic approach to the response latency in routine mental tasks. Paper presented at the Ninth European Mathematical Psychology Group Meeting, Saarbrucken, West Germany, 1977.

Van den Wollenberg, A. L. The Rasch model and time-limit tests. Nijmegen, The Netherlands: Studentenpers, 1979.

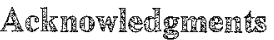

Edw. E. Roskan contributed to the intital developwent of the Poisson-Erlang model for response times. and to shaping the research and analyses based upon ir. The authors thank their colleagues at the University of Nimegen, E. E.Ch.I. Roskan, for reading and commenting upon an earlier version of this paper. and M. M. Nawas, for his editorial assistance.

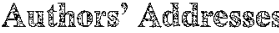

Send requests for reprints or further information to Dr. A. H. G. Sovan der Ven or Dr. J. P. M. Pieters, Department of Mathematical Psychology, Psycholog. ical Laboratory, University of Nijmegen, $\mathbb{B}, \mathrm{O}$. Box 9104, 6500 HE Nijmegen, The Netherlands. 